\title{
THE INFIMUM OF SMALL SUBHARMONIC FUNCTIONS
}

\author{
P. C. FENTON
}

ABSTRACT. Suppose that $u$ is subharmonic in the plane and that, for some $p>1, \lim _{r \rightarrow \infty} B(r) /(\log r)^{p}=\sigma<\infty$. It is shown that, given $\varepsilon>0$,

$$
A(r)>B(r)-(\sigma+\varepsilon) \operatorname{Re}\left\{(\log r)^{p}-(\log r+i \pi)^{p}\right\}
$$

for $r$ outside an exceptional set $E$, where

$$
\lim _{r \rightarrow \infty} \frac{1}{(\log r)^{p-1}} \int_{E \cap[1, r]} \frac{(p-1)(\log t)^{p-2}}{t} d t<\frac{\sigma}{\sigma+\varepsilon} .
$$

1. Introduction. Let $u(z)$ be subharmonic in the plane and define $B(r)=$ $\max _{|z|=r} u(z), A(r)=\inf _{|z|=r} u(z)$. The purpose of this note is to prove

THEOREM. Let $p>1$ be given and suppose that $u(z)$ is subharmonic in the plane and satisfies

$$
\lim _{r \rightarrow \infty} \frac{B(r)}{(\log r)^{p}}=\sigma<\infty
$$

Then, given $\varepsilon>0$,

$$
A(r)>B(r)-(\sigma+\varepsilon) \operatorname{Re}\left\{(\log r)^{p}-(\log r+i \pi)^{p}\right\}
$$

for all $r$ outside a set $E$ such that

$$
\lim _{r \rightarrow \infty} \frac{1}{(\log r)^{p-1}} \int_{E \cap[1, r]} \frac{(p-1)(\log t)^{p-2}}{t} d t<\frac{\sigma}{\sigma+\varepsilon} .
$$

The term $\operatorname{Re}\left\{(\log r)^{p}-(\log r+i \pi)^{p}\right\}$ is $\frac{1}{2} \pi^{2} p(p-1)(\log r)^{p-2}(1+o(1))$ when $r$ is large, and in this form (1.2) should be compared with Theorem 4 of [1], together with Barry's remarks in [1, §7.4]. The inequality is evidently sharp as can be seen from $u(z)=\operatorname{Re}(\log z)^{p}$ (modified slightly in a disc about 0 ). The case $p=1$ in (1.1) is considered separately in $\$ 4$.

If $u(z)$ is subharmonic in the plane then, from the Riesz representation theorem, there exists a unique nonnegative measure $\mu$ defined on all bounded, Borel-measurable subsets of the plane such that, if $R$ is a given positive number,

$$
u(z)=h_{R}(z)+\int_{|\zeta|<R} \log \left|1-\frac{z}{\zeta}\right| d \mu_{\zeta}
$$

for $|z|<R$. Here $h_{R}(z)$ is harmonic in $|z|<R$. Actually to obtain (1.4) it is assumed that $u$ is harmonic at 0 but this may be achieved in the usual way by replacing $u$ in a small disc about 0 by the Poisson integral of its boundary

Received by the editors June 20, 1978 and, in revised form, December 14, 1978.

AMS (MOS) subject classifications (1970). Primary 31A05. 
values on the disc. No loss of generality is entailed since we are concerned with $u(z)$ only when $|z|$ is large. In what follows we shall assume, without loss of generality, that $u(0)=0$.

In [2] Barry has put into subharmonic form results derived by Kjellberg ([4, pp. 190-192]) in the case $u(z)=\log |f(z)|$, where $f$ is an entire function. Some of these are as follows.

Set $\mu^{*}(t)=\mu(|\zeta|<t)$ and define

$$
\begin{gathered}
u_{1}(z, R)=\int_{|\zeta|<R} \log \left|1-\frac{z}{\zeta}\right| d \mu_{\zeta}, \\
u_{2}(z, R)=\int_{|\zeta|<R} \log \left|1+\frac{z}{|\zeta|}\right| d \mu_{\zeta}=\int_{0}^{R} \log \left|1+\frac{z}{t}\right| d \mu^{*}(t),
\end{gathered}
$$

and

$$
u_{3}(z, R)=u(z)-u_{1}(z, R) .
$$

Then, with $B_{j}(r, R)=\max _{|z|=r} u_{j}(z, R), A_{j}(r, R)=\inf _{|z|=r} u_{j}(z, R), j=$ $1,2,3$,

$$
A_{2}(r, R) \leqslant A_{1}(r, R) \leqslant B_{1}(r, R) \leqslant B_{2}(r, R) .
$$

Also

$$
B_{3}(r, R) \leqslant \frac{4 r}{R} B(2 R), \quad A_{3}(r, R) \geqslant-\frac{4 r}{R} B(2 R),
$$

for $0<r<\frac{1}{2} R$. From (1.9) it follows that, for $u(z)$ satisfying $(1.1), u_{1}(z, R)$ converges uniformly to $u(z)$ on bounded sets as $R \rightarrow \infty$ through a sequence.

Finally we note the subharmonic analogue of Jensen's Theorem [3, p. 473]: for $r>0$,

$$
\int_{0}^{r} \log \frac{r}{t} d \mu^{*}(t)=\frac{1}{2 \pi} \int_{0}^{2 \pi} u\left(r e^{i \theta}\right) d \theta<B(r) .
$$

2. A Lemma. We prove the following Lemma which, though very straightforward, is in fact central to the proof of the Theorem.

LemMa. Let $R_{1}, R_{2}$ and $R$ be positive numbers satisfying $R_{1}<R_{2}<R$. Then

$$
I\left(R_{1}, R_{2}, R\right)=\int_{R_{1}}^{R_{2}} \frac{A_{2}(t, R)-B_{2}(t, R)}{t} d t>-\frac{1}{2} \pi^{2} \mu^{*}(R)\left(1+\frac{R_{2}}{R}\right) .
$$

On integrating $z^{-1} \log (1-z / t)$ around a semiannulus in the upper half-plane we obtain, for any positive $t$,

$$
\begin{aligned}
\int_{R_{1}}^{R_{2}}\{\log \mid 1- & \left.\frac{s}{t} \mid-\log \left(1+\frac{s}{t}\right)\right\} \frac{d s}{s} \\
& =\int_{0}^{\pi} \operatorname{Arg}\left(1-\frac{R_{2}}{t} e^{i \theta}\right) d \theta-\int_{0}^{\pi} \operatorname{Arg}\left(1-\frac{R_{1}}{t} e^{i \theta}\right) d \theta .
\end{aligned}
$$

Integrating both sides with respect to $\mu^{*}(t)$ from 0 to $R$ and inverting the order of integration (which is justified since all three integrands are nonposi- 
tive) we obtain

$$
\begin{aligned}
I\left(R_{1}, R_{2}, R\right)= & \int_{0}^{\pi} d \theta \int_{0}^{R} \operatorname{Arg}\left(1-\frac{R_{2}}{t} e^{i \theta}\right) d \mu^{*}(t) \\
& -\int_{0}^{\pi} d \theta \int_{0}^{R} \operatorname{Arg}\left(1-\frac{R_{1}}{t} e^{i \theta}\right) d \mu^{*}(t) \\
= & I_{2}-I_{1} .
\end{aligned}
$$

Integration by parts yields

$$
\begin{aligned}
\int_{0}^{R} \operatorname{Arg}(1- & \left.\frac{R_{2}}{t} e^{i \theta}\right) d \mu^{*}(t) \\
& =\mu^{*}(R) \operatorname{Arg}\left(1-\frac{R_{2}}{R} e^{i \theta}\right)-\int_{0}^{R} \mu^{*}(t) \frac{\partial}{\partial t} \operatorname{Arg}\left(1-\frac{R_{2}}{t} e^{i \theta}\right) d t \\
& =\mu^{*}(R) \operatorname{Arg}\left(1-\frac{R_{2}}{R} e^{i \theta}\right)-\int_{0}^{R} \mu^{*}(t) \frac{R_{2} \sin \theta}{t^{2}+R_{2}^{2}-2 t R_{2} \cos \theta} d t
\end{aligned}
$$

and thus

$$
I_{2}=\mu^{*}(R) \int_{0}^{\pi} \operatorname{Arg}\left(1-\frac{R_{2}}{R} e^{i \theta}\right) d \theta-\int_{0}^{R} \frac{\mu^{*}(t)}{t} \log \left|\frac{t+R_{2}}{t-R_{2}}\right| d t .
$$

There is a similar expression for $I_{1}$.

Now

$$
\operatorname{Arg}\left(1-\frac{R_{2}}{R} e^{i \theta}\right)>-\operatorname{Arcsin} \frac{R_{2}}{R}>-\frac{\pi}{2} \frac{R_{2}}{R}
$$

and also

$$
\begin{aligned}
\int_{0}^{R} \frac{\mu^{*}(t)}{t} \log \left|\frac{t+R_{2}}{t-R_{2}}\right| d t & \leqslant \mu^{*}(R) \int_{0}^{\infty} t^{-1} \log \left|\frac{t+R_{2}}{t-R_{2}}\right| d t \\
& =\mu^{*}(R) \int_{0}^{\infty} t^{-1} \log \left|\frac{t+1}{t-1}\right| d t \\
& =\frac{1}{2} \pi^{2} \mu^{*}(R) .
\end{aligned}
$$

(The value of the integral follows on taking limits as $R_{1} \rightarrow 0$ and $R_{2} \rightarrow \infty$ in (2.2).) Thus

$$
I_{2} \geqslant-\frac{1}{2} \pi^{2} \mu^{*}(R)\left(1+\frac{R_{2}}{R}\right)
$$

On the other hand $I_{1}<0$, and the Lemma follows.

3. Proof of the Theorem. From (1.8), (1.9) and the Lemma we deduce that, for $R_{2}<\frac{1}{2} R$,

$$
\int_{R_{1}}^{R_{2}} \frac{A(t)-B(t)}{t} d t \geqslant-\frac{1}{2} \pi^{2} \mu^{*}(R)\left(1+\frac{R_{2}}{R}\right)-\frac{8 R_{2}}{R} B(2 R) .
$$


Thus, with $\psi(t)=\operatorname{Re}\left\{(\log t)^{p}-(\log t+i \pi)^{p}\right\}$ and $R_{1}=1$,

$$
\begin{aligned}
\int_{1}^{R_{2}} & \frac{A(t)-B(t)+\sigma \psi(t)}{t} d t \\
\geqslant & \frac{\sigma}{p+1} \operatorname{Re}\left\{\left(\log R_{2}\right)^{p+1}-\left(\log R_{2}+i \pi\right)^{p+1}\right\} \\
& -\frac{1}{2} \pi^{2} \mu^{*}(R)\left(1+\frac{R_{2}}{R}\right)-\frac{8 R_{2}}{R} B(2 R)+O(1)
\end{aligned}
$$

We choose $R$ so that the second and third terms of the right-hand side of (3.2) are small. This is done as follows. Given $\eta>0$, we can find arbitrarily large values of $r$ such that

$$
\int_{0}^{r} \frac{\mu^{*}(t)}{t} d t \leqslant B(r) \leqslant(\sigma+\eta)(\log r)^{p} .
$$

Suppose that $\mu^{*}(t)>p(\sigma+2 \eta)(\log t)^{p-1}$ for $r^{\prime} \leqslant t \leqslant r$. Then

$$
(\sigma+2 \eta)\left\{(\log r)^{p}-\left(\log r^{\prime}\right)^{p}\right\} \leqslant \int_{r^{\prime}}^{r} \frac{\mu^{*}(t)}{t} d t \leqslant(\sigma+\eta)(\log r)^{p},
$$

from which it follows that $r^{\prime} \geqslant r^{\nu}$, where $\nu=(\eta /(\sigma+2 \eta))^{1 / p}$. Also

$$
B\left(r^{\prime}\right) \leqslant B(r) \leqslant(\sigma+\eta)(\log r)^{p} \leqslant \frac{(\sigma+\eta)(\sigma+2 \eta)}{\eta}\left(\log r^{\prime}\right)^{p} .
$$

It is thus possible to find arbitrarily large values of $r$ at which

$$
\mu^{*}(r) \leqslant p(\sigma+2 \eta)(\log r)^{p-1} \text { and } B(r) \leqslant \frac{(\sigma+2 \eta)^{2}}{\eta}(\log r)^{p},
$$

and we choose $R$ so that $2 R$ is one such value. Then

$$
\begin{gathered}
\mu^{*}(R) \leqslant \mu^{*}(2 R) \leqslant p(\sigma+2 \eta+o(1))(\log R)^{p-1} \text { and } \\
B(2 R) \leqslant c(\log 2 R)^{p},
\end{gathered}
$$

where $c=(\sigma+2 \eta)^{2} / \eta$.

Returning to (3.2) and making use of (3.3) we have, for $R_{2} \leqslant \frac{1}{2} R$,

$$
\begin{aligned}
J\left(R_{2}\right)= & \int_{1}^{R_{2}} \frac{A(t)-B(t)+\sigma \psi(t)}{t} d t \\
\geqslant & \frac{\sigma}{p+1} \operatorname{Re}\left\{\left(\log R_{2}\right)^{p+1}-\left(\log R_{2}+i \pi\right)^{p+1}\right\} \\
& -\frac{1}{2} \pi^{2} p(\sigma+2 \eta+o(1))(\log R)^{p-1}\left(1+\frac{R_{2}}{R}\right) \\
& -\frac{8 c R_{2}}{R}(\log 2 R)^{p}+O(1) .
\end{aligned}
$$

Now set $R_{2}=R^{1-\alpha}$, where $\alpha>0$ is fixed. Then

$$
\begin{aligned}
J\left(R_{2}\right) \geqslant & \frac{1}{2} \pi^{2} p\left\{\sigma-(\sigma+2 \eta)(1-\alpha)^{1-p}+o(1)\right\}\left(\log R_{2}\right)^{p-1} \\
& -8 c R_{2}^{-\alpha /(1-\alpha)}(1-\alpha)^{-p}\left(\log R_{2}\right)^{p}(1+o(1))+O(1) .
\end{aligned}
$$


Since we may take $\eta>0$ and $\alpha>0$ as small as we please we deduce that

$$
\varlimsup_{r \rightarrow \infty} \frac{J(r)}{(\log r)^{p-1}} \geqslant 0 \text {. }
$$

Suppose now that (1.2) is false for $r$ in a set $E$. Then

$$
\begin{aligned}
J(r) & \leqslant-\varepsilon \int_{E \cap[1, r]} \frac{\psi(t)}{t} d t+\sigma \int_{\sim E \cap[1, r]} \frac{\psi(t)}{t} d t \\
& =-(\sigma+\varepsilon) \int_{E \cap[1, r]} \frac{\psi(t)}{t} d t+\sigma \int_{1}^{r} \frac{\psi(t)}{t} d t .
\end{aligned}
$$

Also $\psi(t)=\frac{1}{2} \pi^{2} p(p-1)(\log t)^{p-2}(1+o(1))$ as $t \rightarrow \infty$ and thus

$$
\begin{aligned}
& \varlimsup_{r \rightarrow \infty} \frac{J(r)}{(\log r)^{p-1}} \\
& \leqslant \frac{1}{2} \pi^{2} p \varlimsup_{r \rightarrow \infty}\left\{\sigma-(\sigma+\varepsilon) \frac{1}{(\log r)^{p-1}} \int_{E \cap[1, r]}(p-1) \frac{(\log t)^{p-2}}{t} d t\right\} .
\end{aligned}
$$

Comparing this with (3.4) we deduce that

$$
\varliminf_{r \rightarrow \infty} \frac{1}{(\log r)^{p-1}} \int_{E \cap[1, r]}(p-1) \frac{(\log t)^{p-2}}{t} d t<\frac{\sigma}{\sigma+\varepsilon} .
$$

This completes the proof of the Theorem.

4. The case $p=1$. When $p=1$ in (1.1) we have on a sequence of $r$

$$
\int_{0}^{r} \frac{\mu^{*}(t)}{t} d t \leqslant B(r)=O(\log r) .
$$

It follows that $\mu^{*}(r)$ is bounded on a sequence and thus bounded (since $\mu^{*}$ is nondecreasing), so that in fact (4.1) holds for all large $r$. We may thus appeal to Theorem 12 of [1] to deduce that, if $h(r)$ is positive and continuous for $r>c>0$ and such that

$$
\int_{c}^{\infty} \frac{h(t)}{t} d t
$$

is divergent, then

$$
A(r)>B(r)-h(r)
$$

for certain arbitrarily large values of $r$. The same result may be obtained from (3.1).

\section{REFERENCES}

1. P. D. Barry, The minimum modulus of small integral and subharmonic functions, Proc. London Math. Soc. 12 (1962), 445-495.

2. On a theorem of Kjellberg, Quart. J. Math. Oxford Ser. (2) 15 (1964), 179-191.

3. W. K. Hayman, The minimum modulus of large integral functions, Proc. London Math. Soc. 2 (1952), 469-512.

4. Bo Kjellberg, On the minimum modulus of entire functions of lower order less than one, Math. Scand. 8 (1960), 189-197.

Department of Mathematics, University of Otago, Otago, New Zealand 